\title{
BMJ Open Efficacy of remdesivir in patients with COVID-19: a protocol for systematic review and meta-analysis of randomised controlled trials
}

\author{
Desye Gebrie (D) , ${ }^{1,2}$ Desalegn Getnet, ${ }^{3}$ Tsegahun Manyazewal (D) ${ }^{2}$
}

To cite: Gebrie $D$, Getnet $D$, Manyazewal T. Efficacy of remdesivir in patients with COVID-19: a protocol for systematic review and meta-analysis of randomised controlled trials. BMJ Open 2020;10:e039159. doi:10.1136/ bmjopen-2020-039159

- Prepublication history and additional material for this paper are available online. To view these files, please visit the journal online (http://dx.doi. org/10.1136/bmjopen-2020039159).

Received 07 April 2020 Revised 13 May 2020 Accepted 20 May 2020

D Check for updates

(c) Author(s) (or their employer(s)) 2020. Re-use permitted under CC BY-NC. No commercial re-use. See rights and permissions. Published by BMJ.

${ }^{1}$ School of Pharmacy, College of Health Sciences, Mekelle University, Mekelle, Ethiopia ${ }^{2}$ Addis Ababa University, College of Health Sciences, Center for Innovative Drug Development and Therapeutic Trials for Africa, Addis Ababa, Ethiopia ${ }^{3}$ Pharmacology and Toxicology Course and Research Team, Department of Pharmacy, College of Health Sciences, Adigrat University, Adigrat, Ethiopia

Correspondence to

Desye Gebrie;

desye.gebrie@mu.edu.et

\section{ABSTRACT}

Background Despite global containment measures to fight the coronavirus disease 2019 (COVID-19), the pandemic continued to rise, rapidly spread across the world, and resulting in 2.6 million confirmed cases and 185061 deaths worldwide as of 23 April 2020. Yet, there are no approved vaccines or drugs to make the disease less deadly, while efforts are underway. Remdesivir, a nucleotide-analogue antiviral drug developed for Ebola, is determined to prevent and stop infections with COVID-19, while results are yet controversial. Here, we aim to conduct a systematic review and meta-analysis of randomised controlled trials (RCTs) to evaluate the efficacy of remdesivir in patients with COVID-19.

Method and analysis We will search MEDLINE-PubMed, Embase, Cochrane Library, ClinicalTrials.gov and Google scholar databases for articles published as of 30 June 2020 and we will complete the study on 30 August 2020 . We will follow the Preferred Reporting Items for Systematic Review and Meta-Analysis Protocols (PRISMA-P) 2015 guidelines for the design and reporting of the results. We will include RCTs that assessed the efficacy of remdesivir versus placebo or standard of care. The primary endpoint will be time to clinical recovery. The secondary endpoints will be proportion of participants relieved from clinical symptoms defined at the time (in hours) from initiation of the study treatment, all-cause mortality, discharged date, frequency of respiratory progression and treatmentemergent adverse events. RevMan V.5.3 software will be used for statistical analysis. Random effects model will be carried out to calculate mean differences for continuous outcome data and risk ratio for dichotomous outcome data between remdesivir and placebo or standard of care.

Ethics and dissemination There are no ethical considerations associated with this study as we will use publicly available data from previously published studies. We plan to publish results in open-access peerreviewed journals and present at international and national conferences.

PROSPERO registration number CRD42020177953.

\section{INTRODUCTION}

Coronavirus diseases 2019 (COVID-19) is caused by a novel $\beta$-coronavirus which is named as SARS-CoV-2. SARS-CoV-2 shares $79 \%$ RNA sequence identity with severe
Strengths and limitations of this study

- This will be the first systematic review and metaanalysis to evaluate the efficacy of remdesivir for COVID-19, which is a newly originated deadly disease.

- Its compliance with the Preferred Reporting Items for Systematic Review and Meta-Analysis for Protocols 2015 (PRISMA-P 2015) will ensure the quality of reporting.

- The use of a combination of multiple electronic databases will include all eligible articles and provide accurate conclusions

- The use of rigorous subgroup and sensitivity analysis will identify possible reasons that may cause significant heterogeneity between studies.

- Its singular focus on one antiviral treatment may preclude decision-making and calls for network meta-analyses once trial results are made available.

acute respiratory syndrome coronavirus (SARS-CoV) and $50 \%$ genomic sequence identity with Middle East respiratory syndrome coronavirus (MERS-CoV) which caused a major outbreak since 2002 and 2012 in China and Saudi Arabia, respectively. ${ }^{1-4}$ Despite global containment measures to fight the disease, the pandemic continued to rise, rapidly spread across the world, and resulting in 2.6 million confirmed cases and 185061 deaths worldwide as of 23 April $2020 .{ }^{5}{ }^{6}$ The outbreak of COVID-19 infection has a significant threat to international health, the economy, psychological stress and mental health worldwide. ${ }^{7-10}$ Yet, there are no approved vaccines or drugs to make the disease less deadly; implying that searching therapeutic options are critical issues to overcome the outbreak. ${ }^{1112}$

Studies are strongly underway to discover rapidly drug candidates for COVID-19, and studies are looking into repurposing drugs that have been used for the treatment of other diseases. As of 29 March 2020, there were 209 
clinical trials registered in ClinicalTrials.gov for COVID-19 therapeutic studies and this number is estimated to go over $500 .{ }^{13}$ Currently, several drugs including remdesivir, hydroxychloroquine, chloroquine, ritonavir+lopinavir, Arbidol and interferon are under randomised controlled trials (RCTs) for efficacy and/or safety evaluations in patients with COVID-19 in different countries. ${ }^{14-19}$ Remdesivir (GS-5734) is among these investigational drugs and some studies reported promising results. ${ }^{19} 20$ Remdesivir is a nucleotide analogue intravenous prodrug developed by Gilead Sciences, an American biopharmaceutical company, for treatment of Ebola virus during the 2014 Ebola outbreak in Western Africa. Remdesivir shows broad-spectrum antiviral activity against many RNA viruses including SARS-CoV-2 through blocking RNA polymerase thereby terminating RNA transcription. A recent study led by the US National Institutes of Health (NIH) that involved two groups of six rhesus macaque experiment monkeys, with one group treated with remdesivir, revealed a significantly lowered COVID-19 disease progression due to remdesivir. ${ }^{21}$ According to a recent report of the US Centers for Disease Control and Prevention (CDC), in vitro and cell culture studies demonstrated broad-spectrum activity of remedesivir against coronavirus. ${ }^{22}$ Nucleoside analogues such as remedesivir can have multiple mechanisms of action, including lethal mutagenesis, obligate or non-obligate chain termination, and perturbation of natural nucleotide triphosphate pools via inhibition of nucleotide biosynthesis. ${ }^{23}{ }^{24} \mathrm{In}$ vitro, remdesivir inhibits all human and animal coronaviruses including SARS-CoV-2, and has shown antiviral and clinical effects in animal models of SARS-CoV-1 and MERS-CoV infections. ${ }^{25-29}$ Remdesivir was among the first treatments used in China as the outbreak emerges and it has been reported as potential treatment options for COVID-19 in the USA, China and Italy. ${ }^{14} 1630$ Following the topline data from the randomised, double-blinded, placebo-controlled trial conducted by National Institute of Allergy and Infectious Diseases (NIAID), ${ }^{31}$ the
US Food and Drug Administration (FDA) has issued an emergency use authorisation (EUA) of the antiviral drug remdesivir for the treatment of patients with COVID-19. ${ }^{32}$ Although clinical trials ${ }^{3133}$ have showed remdesivir as a treatment option for COVID-19, results are controversial. Thus, the proposed systematic review and meta-analysis of RCTs aims to synthesise existing evidence on the efficacy and safety of remdesivir in patients with COVID-19.

\section{METHODS}

We will conduct a systematic review and meta-analysis that will comply with the Preferred Reporting Items for Systematic Review and Meta-Analysis Protocols (PRISMA-P) 2015 guidelines for the design and reporting of the results ${ }^{34}$ (see checklist in online supplementary additional file 1). The protocol has been registered at PROSPERO database, ID: CRD42020177953. ${ }^{35}$

\section{Data sources and searches}

We will search MEDLINE/PubMed (http://www.ncbi. nlm.nih.gov/pubmed/), Embase (http://www.embase. com/), The Cochrane Library (http://www.cochranelibrary.com/), ClinicaTtrials.gov (https://www.clinicaltrials.gov/) and google scholar (https://scholar.google. $\mathrm{com} /$ ) databases for primary articles published as of 30 June 2020 and we will complete the study by 30 August 2020. We will perform hand search from the reference lists of a key articles to identify eligible RCTs and supplement the searching. We will include all potential RCTs that evaluated the efficacy of remdesivir versus placebo or standard of care in patients with COVID-19 with no limitations on the geographical location of studies but published in English language. We will do a rigorous search strategy using the key words including 2019 novel coronavirus, 2019-nCov, coronavirus disease 2019, COVID-19, SARS-cov-2, severe acute respiratory syndrome-coronavirus-2, remdesivir, GS-5734, nucleotideanalogue, antiviral agents, randomized controlled trials,

Table 1 Search strategy for the MEDLINE-PubMed database

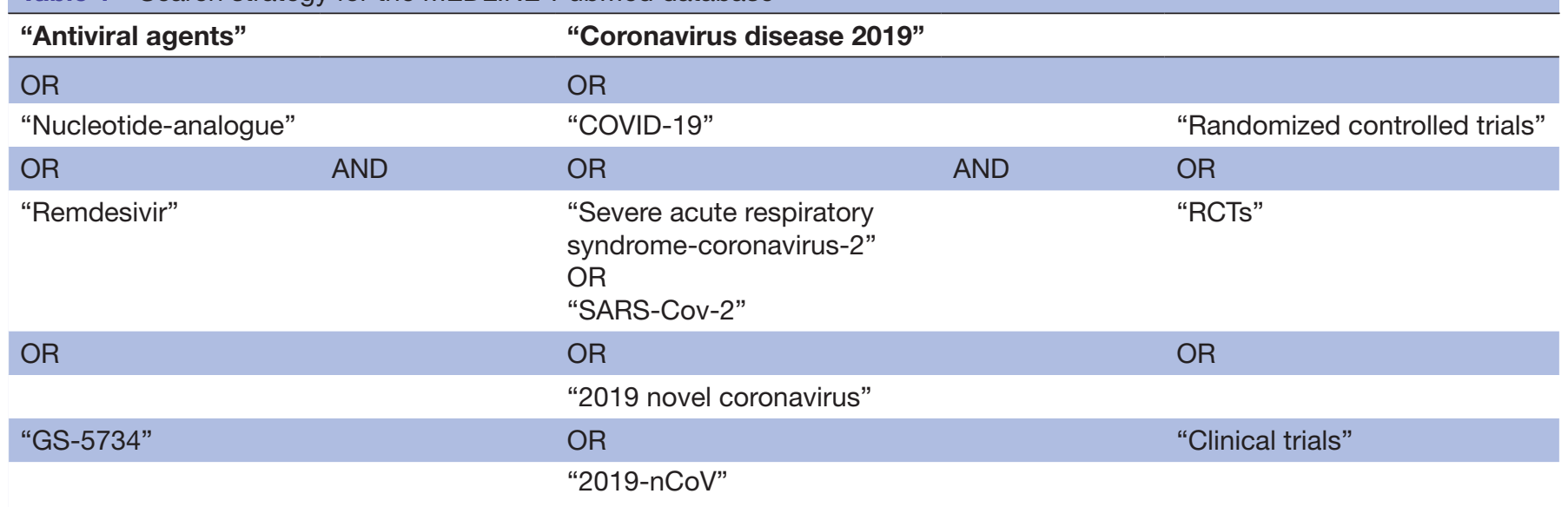


clinical trials and RCTs. Table 1 summarises the search strategy that we will applied in PubMed database, while details of this strategy that we will also adapt for other databases searches are described in online supplementary additional file 2 (table 1 ).

\section{Eligibility criteria}

We will formulate our participant's eligibility criteria using PICOS (participants, interventions, comparison, outcomes, and study designs) description model. ${ }^{36}$

- Participants.

- Patients with confirmed COVID-19.

- Men and/or women of any age.

- At any clinical stage of the disease, thus mild, moderate or severe/critical case.

- With or without other comorbid conditions.

Intervention.

- Remdesivir of any dose.

- Comparator.

- Remdesivir placebo or standard of care.

- Outcomes/endpoints.

- Primary endpoints.

- Time to clinical recovery.

- Secondary endpoints.
- Proportion of participants relieved from clinical symptoms defined at the time (in hours) from initiation of the study treatment.

- All-cause mortality.

- Discharged date.

- Frequency of respiratory progression.

- Oxygen saturation.

- Treatment-emergent adverse events.

Study design.

- Only RCTs evaluating the efficacy of remdesivir versus placebo or standard of care in patients with COVID-19.

\section{Study selection}

All the retrieved papers will be transferred to EnDnote 7 and duplicates will be removed. Two investigators will independently assess the title and abstract of all the retrieved papers based on the eligibility criteria. The two investigators will independently evaluate the full texts. Disagreements between the two investigators will be settled through discussion, and if persisted, the third investigator will be involved as arbitrator. Figure 1 summarises the design that we will use to report the study result in line with the PRISMA-P 2015 guidelines (figure 1).

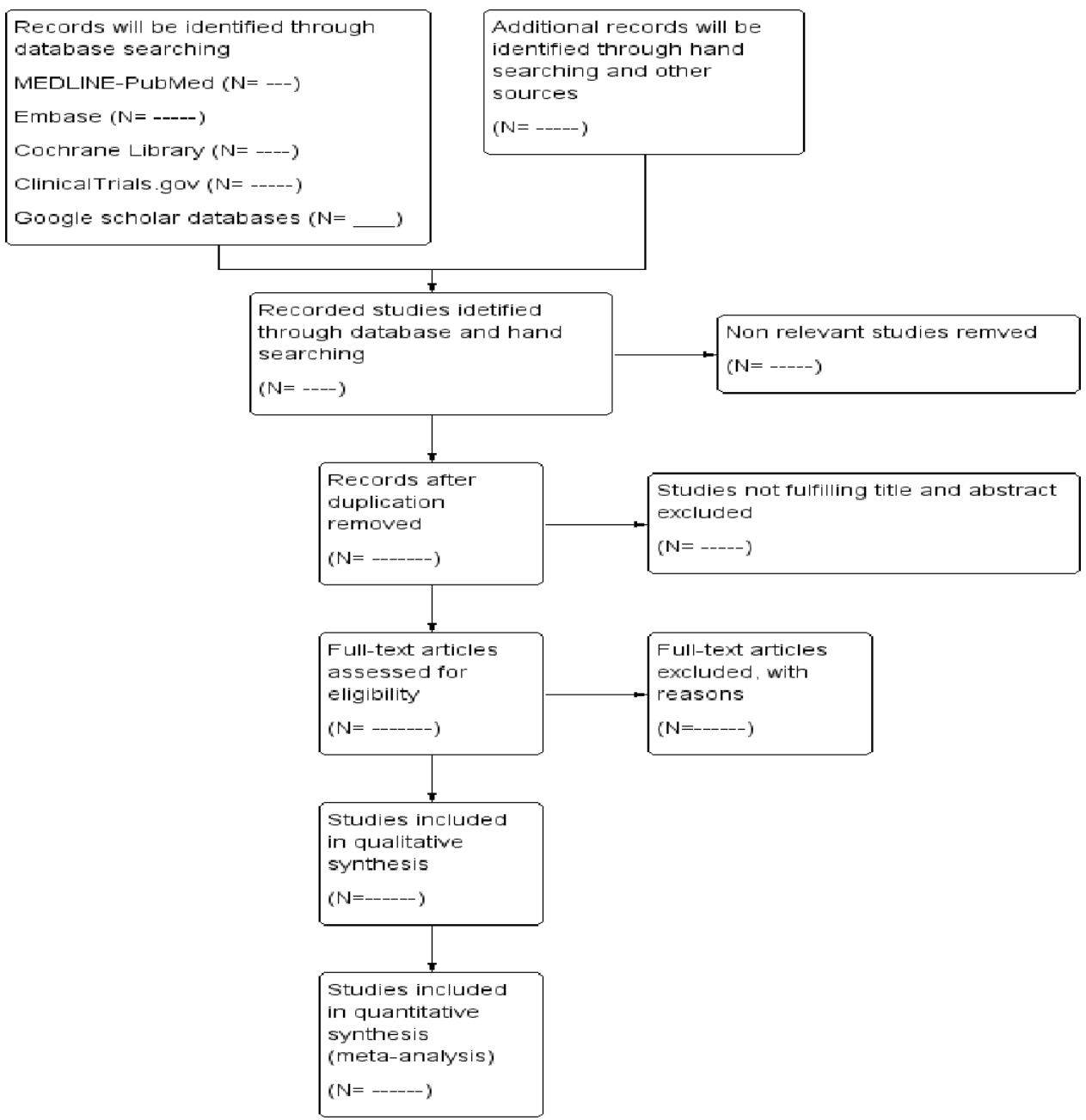

Figure 1 Preferred Reporting Items for Systematic Review and Meta-Analysis Protocols flow diagram of the study. 


\section{Data extraction}

Two authors will independently extract data according to the predesigned data extraction tool. The following data will be extracted from each included RCTs.

- First author.

- Year of publication.

- Study country.

- Funding information.

- Patient characteristics (mean age of the participant, sex, comorbid conditions, number of comorbidities, symptom severity).

- Interventions (remdesivir, dose of remdesivir and route of administration).

- Comparators (remdesivir placebo, standard of care).

- Number of participants randomised in each group.

- Treatment follow-up period.

- Outcomes (primary, secondary and other outcomes).

\section{Assessment of risk of bias}

The Cochrane risk of bias tool ${ }^{37}$ will be used to assess the risk of bias for each included study. The risk of bias of each trial will be judged by two independent investigators as 'Low', 'Some concerns' or 'High' based on the critical domains, including bias arising from the randomisation process, bias due to deviations from intended interventions, bias due to missing outcome data, bias in measurement of the outcome and bias in selection of the reported result. Disagreements will be resolved by discussion among the two investigators. If the disagreements persist, the third investigator will chip in as an arbitrator.

\section{Statistical analysis}

All statistical analyses will be carried out using the computer software packages RevMan V.5.3. ${ }^{38}$ Mean differences (MDs) with $95 \%$ CIs will be used to measure the effects of treatment for continuous outcome data. We will convert other forms of data into MDs using standard conversion formula. For outcome variables reported in different scales, we will use standard mean differences with $95 \%$ CIs. The treatment effect of binary outcome data will be summarised using risk ratios (RRs) with $95 \%$ CIs. Other binary outcome data will be converted into RRs. Mantel-Haenszel method ${ }^{39}$ will be used to pool effect estimates of dichotomous outcomes and inverse variance for continuous outcomes. Cochrane $Q$ test $^{40}$ will be used to assess heterogeneity between studies, and $\mathrm{I}^{2}$ testing ${ }^{41}$ will be done to quantify heterogeneity between studies, with values $>50 \%$ representing moderate-to-high heterogeneity. A random effects model will be used to pool the data. $^{42}$ Subgroup analysis will be carried out between studies with different duration of follow-up, age of participants, severity of the disease, comorbidities, settings and quality of studies for risk of bias. Following the subgroup analysis, we will look at the data for heterogeneity, and if acceptable, we will perform a meta-analysis. If the data are heterogeneous, we will do a narrative description of findings. To see the robustness of pooled data, sensitivity analysis will be conducted between low and high risk of bias, and with or without biassed studies. We will use the GRADEprofiler software from Cochrane Systematic Reviews to assess the quality of evidence per outcome and ultimately to create a summary of findings table and evidence profile. All statistical analysis with a $p$ value $<0.05$ will be considered statistically significant.

\section{Addressing missing data}

When individual participant's data are initially unavailable, we will review the original source, and/or published trial reports, and we will contact the authors to obtain clarification for these data.

\section{Reporting bias}

We will conduct funnel plot and Egger test to check any possible reporting bias if a sufficient number of included studies (at least 10 trials) are available in this study. ${ }^{43}$

\section{Patient and public involvement}

Patients and public will not be involved in this systematic review and meta-analysis. However, once our findings are disseminated, it will be shared through social networks.

\section{Ethics and dissemination}

There are no ethical considerations associated with this study as we will use publicly available data from previously published studies. We plan to publish results in openaccess peer-reviewed journals and present at international and national conferences.

\section{Amendments}

The protocol for this study will be amended as necessary.

Acknowledgements The authors would like to acknowledge the Center for Innovative Drug Development and Therapeutic Trials for Africa (CDT-Africa), College of Health Sciences, Addis Ababa University which funds this study.

Contributors DGeb conceived the study, developed the study criteria, searched the literature, wrote the protocol and drafting the manuscript. DGet conducted the preliminary search and TM copyedited and revised the manuscript. All authors have read and approved the manuscript.

Funding This study is supported by Centre for Innovative Drug Development and Therapeutic Trials for Africa (CDT-Africa), College of Health Sciences, Addis Ababa University.

Competing interests All review authors declare that they have no competing interests. The funder has no role in the design, syntheses and report of the study.

Patient consent for publication Not required.

Provenance and peer review Not commissioned; externally peer reviewed.

Open access This is an open access article distributed in accordance with the Creative Commons Attribution Non Commercial (CC BY-NC 4.0) license, which permits others to distribute, remix, adapt, build upon this work non-commercially, and license their derivative works on different terms, provided the original work is properly cited, appropriate credit is given, any changes made indicated, and the use is non-commercial. See: http://creativecommons.org/licenses/by-nc/4.0/.

\section{ORCID iDs}

Desye Gebrie http://orcid.org/0000-0002-5137-6791

Tsegahun Manyazewal http://orcid.org/0000-0002-8360-7574

\section{REFERENCES}

1 Wu F, Zhao S, Yu B, et al. Complete genome characterization of a novel coronavirus associated with severe human respiratory disease in Wuhan, China. bioRxiv 2020. 
2 Zhou P, Yang XL, Wang XG, et al. Discovery of a novel coronavirus associated with the recent pneumonia outbreak in humans and its potential bat origin. bioRxiv 2020.

3 Lu R, Zhao X, Li J, et al. Genomic characterisation and epidemiology of 2019 novel coronavirus: implications for virus origins and receptor binding. Lancet 2020;395:565-74

4 Morse JS, Lalonde T, Xu S, et al. Learning from the past: possible urgent prevention and treatment options for severe acute respiratory infections caused by 2019-nCoV. Chembiochem 2020;21:730-8.

5 Sohrabi C, Alsafi Z, O'Neill N, et al. World Health organization declares global emergency: a review of the 2019 novel coronavirus (COVID-19). Int J Surg 2020;76:71-6.

6 Worldometer. Coronavirus update (live). Available: https://www. worldometers.info/coronavirus/

7 Zhang $\mathrm{C}$, Huang S, Zheng $\mathrm{F}$, et al. Controversial treatments: an updated understanding of the coronavirus disease 2019. J Med Virol 2020;9.

8 Afonso P. The Impact of the COVID-19 Pandemic on Mental Health]. Acta Med Port 2020.

9 Torales J, O'Higgins M, Castaldelli-Maia JM, et al. The outbreak of COVID-19 coronavirus and its impact on global mental health. Int $J$ Soc Psychiatry 2020;20764020915212:002076402091521.

10 Hiremath P, Suhas Kowshik CS, Manjunath M, et al. COVID 19: impact of lock-down on mental health and tips to overcome. Asian $J$ Psychiatr 2020;51:102088.

11 Lu H. Drug treatment options for the 2019-new coronavirus (2019nCoV). Biosci Trends 2020;14:69-71.

$12 \mathrm{Li} \mathrm{G}$, De Clercq E. Therapeutic options for the 2019 novel coronavirus (2019-nCoV), 202019:149-150. Available: www.nature. com

13 US National Library of Medicine. Clinical trials registry, 2020. Available: https://clinicaltrials.gov/ct2/results?term $=$ covid+19+\& Search=Search [Accessed 29 Mar 2020].

14 Center for disease control and prevention. Information for clinicians on therapeutic options for COVID-19 patients, 2020. Available: https://www.cdc.gov/coronavirus/2019-ncov/hcp/therapeuticoptions.html [Accessed 21 Mar 2020].

15 The First Affiliated Hospital Zhejiang University School of Medicine. Handbook of COVID-19 prevention and treatment. Hand Book 2020.

$16 \mathrm{Li} \mathrm{H}$, Wang YM, Xu JY, et al. [Potential antiviral therapeutics for 2019 Novel Coronavirus]. Zhonghua Jie He He Hu Xi Za Zhi 2020;43:E002-E.

17 Paules $\mathrm{Cl}$, Marston HD, Fauci AS. Coronavirus Infections-More than just the common cold. JAMA 2020. doi:10.1001/jama.2020.0757. [Epub ahead of print: 23 Jan 2020].

18 Devlin H, Sample I. Hopes rise over experimental drug's effectiveness against coronavirus. The Guardian 2020.

19 Dong L, Hu S, Gao J. Discovering drugs to treat coronavirus disease 2019 (COVID-19). Drug Discov Ther 2020;14:58-60.

20 Chan KW, Wong VT, Tang SCW. COVID-19: an update on the epidemiological, clinical, preventive and therapeutic evidence and guidelines of integrative Chinese-Western medicine for the management of 2019 novel coronavirus disease. Am J Chin Med 2020;48:737-62.

21 U.S. National Institutes of Health (NIH). Antiviral remdesivir prevents disease progression in monkeys with COVID-19: study supports clinical testing under way across U.S. news release, 2020. Available: https://www.nih.gov/news-events/news-releases/antiviral-remdesivirprevents-disease-progression-monkeys-covid-19 [Accessed $17 \mathrm{Apr}$ 2020].

22 U.S centers for disease control and prevention (CDC). information for clinicians on investigational therapeutics for patients with COVID-19. CDC, 2020. Available: https://www.cdc.gov/coronavirus/2019-ncov/ hcp/therapeutic-options.html [Accessed 13 Apr 2020].
23 Agostini ML, Andres EL, Sims AC, et al. Coronavirus susceptibility to the antiviral remdesivir (gs-5734) is mediated by the viral polymerase and the proofreading exoribonuclease. $m B i o$ 2018;9:e00221-18.

24 Guo Y-R, Cao Q-D, Hong Z-S, et al. The origin, transmission and clinical therapies on coronavirus disease 2019 (COVID-19) outbreak an update on the status. Mil Med Res 2020;7:11.

25 Sheahan TP, Sims AC, Graham RL, et al. Broad-Spectrum antiviral GS-5734 inhibits both epidemic and zoonotic coronaviruses. Sci Trans/ Med 2017;9:eaal3653.

26 Warren TK, Jordan R, Lo MK, et al. Therapeutic efficacy of the small molecule GS-5734 against Ebola virus in rhesus monkeys. Nature 2016;531:381-5.

27 Brown AJ, Won JJ, Graham RL, et al. Broad spectrum antiviral remdesivir inhibits human endemic and zoonotic deltacoronaviruses with a highly divergent RNA dependent RNA polymerase. Antiviral Res 2019;169:104541.

28 Sheahan TP, Sims AC, Leist SR, et al. Comparative therapeutic efficacy of remdesivir and combination lopinavir, ritonavir, and interferon beta against MERS-CoV. Nat Commun 2020;11:222.

29 de Wit E, Feldmann F, Cronin J, et al. Prophylactic and therapeutic remdesivir (GS-5734) treatment in the rhesus macaque model of MERS-CoV infection. Proc Natl Acad Sci U S A 2020;117:6771-6.

30 Holshue ML, DeBolt C, Lindquist S, et al. First case of 2019 novel coronavirus in the United States. N Engl J Med 2020.

31 National Institute of Allergy and Infectious Diseases. Nih clinical trial shows Remdesivir accelerates recovery from advanced COVID-19, 2020. Available: https://www.niaid.nih.gov/news-events/nih-clinicaltrial-shows-remdesivir-accelerates-recovery-advanced-covid-19 [Accessed 19 Apr 2020].

32 US Food and Drug Administration. Remdesivir EUA letter of authorization, 2020. Available: https://www.fda.gov/media/137564/ download [Accessed 1 May 2020].

33 Wang $Y$, Zhang D, Du G, et al. Remdesivir in adults with severe COVID-19: a randomised, double-blind, placebo-controlled, multicentre trial. Lancet 2020;395:1569-78.

34 Shamseer L, Moher D, Clarke M, et al. Preferred reporting items for systematic review and meta-analysis protocols (PRISMA-P) 2015 elaboration and explanation. BMJ 2015;350:g7647.

35 Gebrie D, Getnet D, Manyazewal T. Efficacy of remdesivir versus placebo for the treatment of coronavirus diseases 2019 (COVID-19): a protocol for systematic review and meta-analysis of randomized controlled trials, 2020. Available: https://www.crd.york.ac.uk/ prospero/display_record.php?ID=CRD42020177953

36 Guyatt GH, Oxman AD, Kunz R, et al. Grade guidelines: 2. framing the question and deciding on important outcomes. J Clin Epidemiol 2011:64:395-400.

37 Higgins JPT. Cochrane Handbook for systematic reviews of interventions. 2nd ed. John Wiley \& Sons, 2019.

38 Review Manager (RevMan) [Computer program]. Version 5.3. Copenhagen: The Nordic Cochrane Centre, The Cochrane Collaboration, 2014

39 Suesse T, Liu I. Mantel-Haenszel estimators of a common odds ratio for multiple response data. Stat Methods Appt 2019;28:57-76.

40 Higgins JPT, Thompson SG, Deeks JJ, et al. Measuring inconsistency in meta-analyses. BMJ 2003;327:557-60.

41 Higgins JPT, Thompson SG. Quantifying heterogeneity in a metaanalysis. Stat Med 2002;21:1539-58.

42 Bell A, Fairbrother M, Jones K. Fixed and random effects models: making an informed choice. Qual Quant 2019;53:1051-74.

43 Sterne JAC, Egger M, Moher D. Chapter 10: Addressing reporting biases. 2011. In: Cochrane Handbook for Systematic Reviews of Interventions Version 510 (updated March 2011) [Internet. The Cochrane Collaboration, 2011. www.cochrane-handbook.org 